\title{
African Trypanosomiasis
}

National Cancer Institute

\section{Source}

National Cancer Institute. African Trypanosomiasis. NCI Thesaurus. Code C84541.

A parasitic disorder caused by protozoa of the Trypanosoma brucei species. It is transmitted by flies and is endemic in various regions of Sub-Saharan Africa. Signs and symptoms include fever, joint pain, headache, and significant swelling of the lymph nodes. If left untreated, the parasitic infection causes anemia, heart, kidney, and endocrine failure, and neurologic damage. Subsequently patients develop confusion, disruption of the sleep cycle, and mental deterioration. The infection may lead to coma and death. 